\title{
Long-term durability of resection and end-to-end anastomosis for ascending aortic aneurysms
}

\author{
Massimo Massetti, MDa \\ Sebastien Veron, MD ${ }^{\mathrm{a}}$ \\ Eugenio Neri, $M D^{b}$ \\ Olivier Coffin, $\mathrm{MD}^{\mathrm{a}}$ \\ Olivier le Page, $M^{\mathrm{a}}$ \\ Gerard Babatasi, MD, $\mathrm{PhD}^{\mathrm{a}}$ \\ Dimitrios Buklas, MDa \\ Dominique Maiza, $\mathrm{MD}^{\mathrm{a}}$ \\ Jean Louis Gerard, MD \\ Andre Khayat, $\mathrm{MD}^{\mathrm{a}}$
}

Background: Ascending aortic aneurysms with normal sized sinotubular junction are generally treated by resection of the dilated aorta and replacement with tubular graft. Aortic resection and direct end-to-end anastomosis has been applied to repair aortic coarctation, interrupted aortic arch, and traumatic aortic rupture. No data exist regarding the long-term durability of this approach in ascending aortic aneurysms. The aim of this case-control study was to illustrate the durability of this operation by presenting our entire experience and the long-term follow up of a cohort of 34 patients who underwent ascending aortic aneurysm resection and primary end-toend anastomosis between January 1990 and March 2003 in Caen University Hospital (Caen, France).

Methods: The mean age of patients was $61.5 \pm 12.5$ years, and there were 18 male and 16 female patients. The operative technique included extensive mobilization of the arch, supra-aortic trunks, and inferior vena cava to enable approximation of the aortic ends, thus avoiding tension on the suture lines. Associated aortic valve replacement was performed in 27 patients; mechanical valves were used in 19. A bicuspid aortic valve was present in 9 patients; in 3 cases the valve was regurgitant. Aortic valve regurgitation was present in a total of 7 patients. Patients were followed up at regular intervals; total follow-up was 2187 patient-months, with a median follow-up time of 72 months per patient (25th-75th percentile 10.5-102.7 months).

Results: One patient died 10 days after the operation of aortic rupture related to suture infection caused by mediastinitis. Late deaths occurred in 3 patients, who died 12, 62, and 71 months after the operation, but none of these deaths were attributable to late aortic repair failure. No patient in this series required reoperation, including patients with aortic regurgitation or bicuspid aortic valve. Follow-up was 91.1\% complete at the closing date of April 1, 2003. The Kaplan-Meier estimate of survival for all patients was 120.4 months (95\% confidence interval 105.1-135.7 months). The median of preoperative maximal aortic diameter was $55.1 \mathrm{~mm}$ (range 50.3 to $67.5 \mathrm{~mm}, 25$ th-75th percentile $50.5-56.8 \mathrm{~mm}$ ). The median immediate postoperative diameter was $40.3 \mathrm{~mm}$ (range $33.4-46.4 \mathrm{~mm}$, 25th-75th percentile 37.2-42.0 mm, $P<.0001$ relative to preoperative diameter), and the median length of the resected aortic segment was $52 \mathrm{~mm}$ (range $48-76 \mathrm{~mm}$, 25th-75th percentile 50.1-66.4 mm). The median decrease of aortic diameter was $24.9 \mathrm{~mm}$ (range 8.9-32.6 mm, 25th-75th percentile 18.2-26.6 mm).The median aortic diameter at the end of the follow-up was $41.0 \mathrm{~mm}$ (range 34.6-46.1 mm, 25th-75th percentile $37.0-43.2 \mathrm{~mm}, P=.6$ relative to immediate postoperative diameter). 


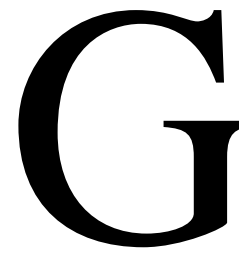

raft interposition after aneurysmal resection is the conventional technique to treat an ascending aortic aneurysm. Reduction aortoplasty, with or without external prosthetic support, represents an alternative approach in selected cases. Aneurysmal excision without graft prosthesis represents a third alternative approach to treat a segmental dilation of the proximal aorta. Aortic resection and direct anastomosis has been considered as standard treatment for certain congenital anomalies, such as coarctation and interrupted aortic arch. Primary repair has also been used occasionally in traumatic aortic rupture ${ }^{1}$ and in special cases of ascending aortic aneurysm ${ }^{2,3}$ or dissection. ${ }^{4}$ With regard to acute aortic dissection, current evidence indicates that this strategy does not provide durable results, ${ }^{5}$ whereas no data exist regarding the long-term durability of this approach for ascending and descending thoracic aortic aneurysms. We report here the long-term follow-up of patients with ascending aortic aneurysm treated with aneurysmal resection and direct anastomosis. We also describe the technique used to relieve tension at the anastomosis, which enables the surgeon to perform primary end-to-end aortic anastomosis for ascending aneurysm in young patients as well in the older population with less mobile and elastic aortas.

\section{Patients and Methods}

Between January 1990 and March 2003, a total of 34 patients were treated at Caen University Hospital with the technique of ascending aortic aneurysm resection and primary end-to-end anastomosis. There were 16 women and 18 men, and the mean age was 61.5 years (median 65.2 years, range 25-78 years). Bicuspid aortic valves were present in 9 patients, and 3 of these valves were regurgitant. Aortic valve regurgitation was present in 7 patients. Twenty-seven of these patients underwent associated aortic valve replacement, 5 for aortic regurgitation and 22 for aortic stenosis; mechanical valves were used in 19 cases. Repair of aortic leaflet prolapse, which was causing severe aortic valve regurgitation, was performed in 2 cases.

Table 1 illustrates in detail the clinical characteristics of all patients. The severity of histologic abnormalities of the resected aortic wall slides is expressed by the aortic wall score according to Matthias Bechtel and colleagues. ${ }^{6}$

\section{Follow-up and Measurement Criteria}

A combination of follow-up methods was used simultaneously to provide the most complete information possible. All patients were followed up by their cardiologist and general practitioner; moreover, the common closing data method was used in April 2003.

During postoperative office visits, each patient was seen by the cardiologist. Transthoracic or transesophageal echocardiography was performed. Mitral valve was systematically explored during follow-up. Chest computed tomographic (CT) scan was obtained for all patients at the follow-up closing time by April 2003.

All living patients (30/34 patients) could be traced at the common closing date and contacted by telephone; a total of 28 patients agreed to undergo clinical and instrumental investigations. Two patients refused to join clinical controls and to undergo CT scan study; nonetheless, for them recent CT scan data $(<9$ months previously) were available. Total follow-up was 2187 patientmonths, with a median follow-up of 72 months per patient (25th75th percentile 10.5-102.7 months). Preoperative measurements of the ascending aorta diameters were performed on CT scan; furthermore the aorta was measured intraoperatively before and after resection. The extent of resection was measured directly from the resected aorta specimen. During the follow-up, aortic diameters were measured on the chest CT scan.

Because of the extensive aortic resection, resulting in a marked proximity between the sinotubular junction and the origin of the brachiocephalic trunk resection, to guarantee an adequate comparison of measurements aortic size was assayed about $10 \mathrm{~mm}$ above the sinotubular junction, either before or after the procedure. For graphic representation of the mean aortic root diameters at followup, a moving band method was used. ${ }^{7}$

\section{Operative Technique}

The operation was performed through a median sternotomy with aortoatrial cannulation for the extracorporeal circulation under general moderate hypothermia; cold blood cardioplegia was used for myocardial protection. The aortic cannula was placed in the proximal transverse aortic arch. With the pump on, the heart was decompressed, and the aorta was then completely mobilized from the surrounding structures. The procedure entailed approximation of the aortic arch toward the valve level (Figure 1, $A-D$ ); for this purpose, with the goal of relieving tension at the anastomosis level, the horizontal aorta was largely mobilized, mainly (1) by dissecting the first portion of the supra-aortic trunks and (2) by dissecting the pericardial reflections adjacent to the inferior vena cava and left atrium. Both aortic arch and the proximal aorta were in this way completely suitable to be approximated without tension after the aneurysmal resection. The ascending aorta was then crossclamped distal to the aneurysm and opened. The aneurysm was resected between two circumferential aortotomies: the first at the level of sinotubular junction and the second at the distal edge of the aneurysm. Cardioplegia was then infused directly into the coronary ostia according to the target $\left(10^{\circ} \mathrm{C}\right)$ myocardial temper- 
ature (as monitored by an intramyocardial temperature probe). When indicated, the aortic valve surgery was performed without any modification of the surgical technique. The two ends of the aorta were finally sutured with a continuous 4-0 polypropylene suture. After standard deairing procedures, the aortic crossclamp was released. Additional dissection of the aortic arch to avoid tension was rarely necessary.

\section{Statistical Analysis}

Because of to the small sample, distribution-free methods based on permutation inference were used to avoid unrealistic functional assumptions. ${ }^{8}$ Statistical routines, implemented the StatXact statistical packages (Cytel Software Corporation, Cambridge, Mass), were used. Categoric variables were tested with the Fisher exact test; continuous variables were compared with both permutation procedures on unpaired data and permutation procedures on paired data, as appropriate. A permutation procedure for repeated measures was used to test differences of aortic diameters before and after the procedure during the follow up. For graphic representation of the mean aortic root diameters at follow-up, a moving band method was used. ${ }^{7}$ This method was chosen because of the variable follow-up period. With this method the mean follow-up time and the mean diameter were calculated for the 5 patients with the shortest follow-up period. Then the band moved in time, incorporating the sixth patient and excluding the first patient. For these patients a mean follow-up period and a mean diameter were calculated again. This was repeated until the 5 patients with the longest follow-up period were included. The width of the band thus consisted of five patients and varied with time. So that the individual behavior of the aortic diameters could be clarified, the diameter modification was shown for each patient with at least three measurements. Survival data were analyzed with standard Kaplan-Meier actuarial techniques for estimation of survival probabilities and compared with log-rank tests.

\section{Results}

\section{Patients}

One patient died 10 days after the operation of aortic rupture related to suture infection caused by mediastinitis. Late deaths occurred in 3 patients, who died respectively 12, 62, and 71 months after the operation of lung cancer, car crash, and respiratory failure. No patient had neurologic deficits. No patient in this series required reoperation, including patients with aortic regurgitation or bicuspid aortic valve. The Kaplan-Meier estimate of survival for all patients was 120.4 months (95\% confidence interval 105.1-135.7 months), with a freedom from death of $83.9 \%$ (95\% confidence interval 61.9\%-93.8\%) at 132 months (Figure 2).

\section{Diameters}

Aortic diameter measurements before and after cardiopulmonary bypass (CPB) were available for all 34 patients. The mean values of aortic measurements before and at different time points after the operation are shown in Figures 3 and 4. The individual increases of aortic diameters of patients with 3 or more measurements available are presented in the
TABLE 1. Clinical, operative, and perioperative features (n $=34$ patients)

Aortic stenosis, severe (No.)

Aortic stenosis, moderate to severe (No.)

Aortic regurgitation, severe (No.)

Ascending aorta aneurysm without aortic valve disease (No.)

Mitral regurgitation (No.)

Redo surgery (No.)

Bicuspid aortic valve (No.)

Ascending aortic aneurysm (No.)

Inherited connective tissue diseases (No.)

Aortic score (median with 25th-75th percentile)

New York Heart Association functional class (No.)

1

2

3

4

Canadian Cardiovascular Society (No.)

1

2

3

Associated procedures (No.)

Aortic valve replacement (No.)

Aortic valve repair (No.)

Coronary artery bypass grafting (No.)

Transfusion performed (No.)

Units transfused (median with 25th-75th percentile)

CPB time (min, median with 25th-75th percentile)

Crossclamp time (min, median with 25th75th percentile)

Intensive care unit time (h, median with 25th-75th percentile)

Postoperative hospital stay (d, median with 25th-75th percentile)

$14(41.1 \%)$

$13(38.2 \%)$

$5(14.7 \%)$

$2(5.8 \%)$

$26(76.4 \%)$

$6(17.6 \%)$

$2(5.8 \%)$

$27(79.4 \%)$

$2(5.8 \%)$

$0(0 \%)$

$14(41.1 \%)$

$1.3(1-2)$

$85(74-109)$

$61(53-76)$

$11(15-2)$

$10(8-12)$

Figure 4. There was a statistically significant difference between the mean aortic diameter before and directly after $\mathrm{CPB}$. The median preoperative maximal aortic diameter was $55.1 \mathrm{~mm}$ (range: $50.3-67.5 \mathrm{~mm}, 25$ th-75th percentile 50.5-56.8 mm); the median immediate postoperative diameter was $40.3 \mathrm{~mm}$ (range 33.4-46.4 mm, 25th-75th percentile $37.2-42.0 \mathrm{~mm}, P<.0001$ relative to preoperative diameter), and the median length of the resected aortic segment was $52 \mathrm{~mm}$ (range 48 to $76 \mathrm{~mm}, 25$ th-75th percentile 50.1-66.4 mm). The median decrease in aortic diameter was $24.9 \mathrm{~mm}$ (range 8.9-32.6 mm, 25th-75th percentile 18.2$26.6 \mathrm{~mm})$.

At follow-up, the mean aortic diameters showed no statistically significant enlargement when compared with the findings of aortic contrast CT scan at discharge, with a median aortic diameter at the end of the follow-up of 41.0 $\mathrm{mm}$ (range 34.6-46.1 mm, 25th-75th percentile 37.0-43.2 


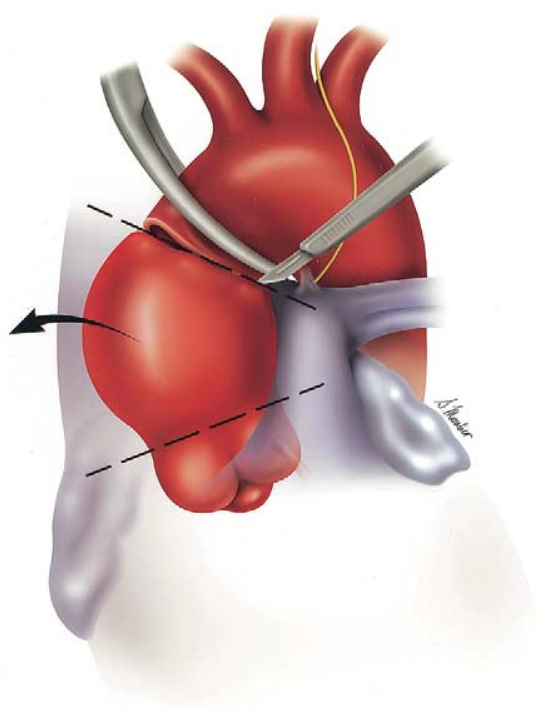

A

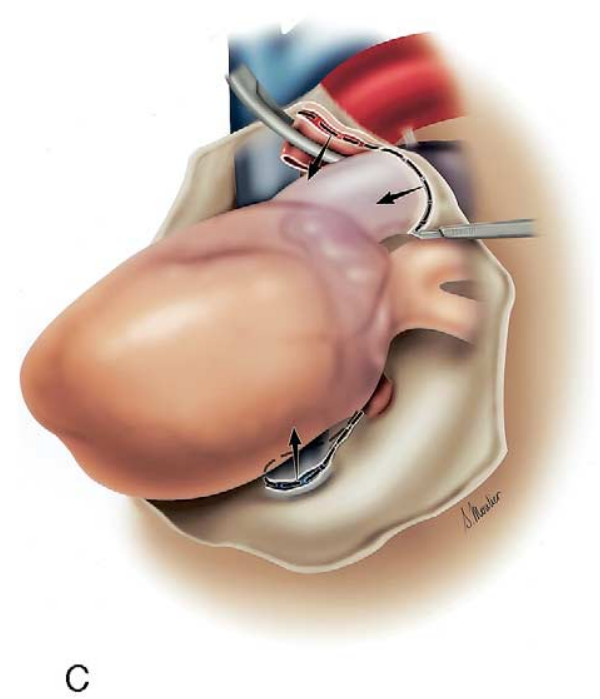

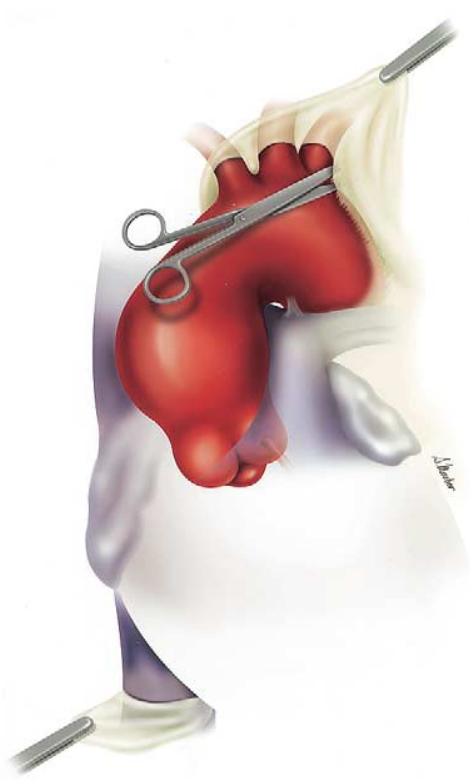

B

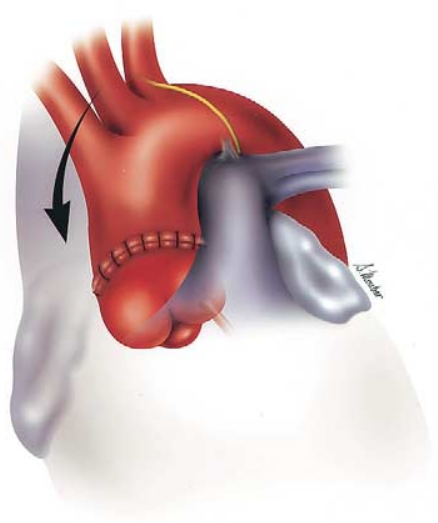

Figure 1. Ascending aorta is crossclamped distal to aneurysm and opened. Aneurysm is resected between two circumferential aortotomies: first at level of sinotubular junction and second at distal edge of aneurysm (A). Horizontal aorta is then largely mobilized, mainly by dissecting first portion of supra-aortic trunks (B) and by dissecting pericardial reflections adjacent to inferior vena cava and left atrium (C). This part of operation can be performed before aortic crossclamping. Two ends of aorta, without tension, are finally sutured with a continuous 4-0 polypropylene suture (D).

$\mathrm{mm}, P=.6$ relative to immediate postoperative diameter). With regard to aortic score, we found no difference between patients with aortic regurgitation and those with stenosis (median difference of 0,2 -sided $P=.57$ ).

\section{Discussion}

With the exception of a few conditions, in which a genetic disorder of collagen structure has been identified, pathophysiology of ascending aortic dilatation remains obscure.
In particular the complex relationships among mechanical stress, medial degeneration, and genetic factors ${ }^{9-12}$ leave open the debate about the strategies of prevention and timing, as well as the surgical techniques to be used in this condition.

Only with regard to the Marfan syndrome and other inherited connective tissue disorders has a consensus been reached that more radical operations, extended to the aortic root can be performed. For lesions attributable to other causes, the surgical indications and the choice of the pro- 


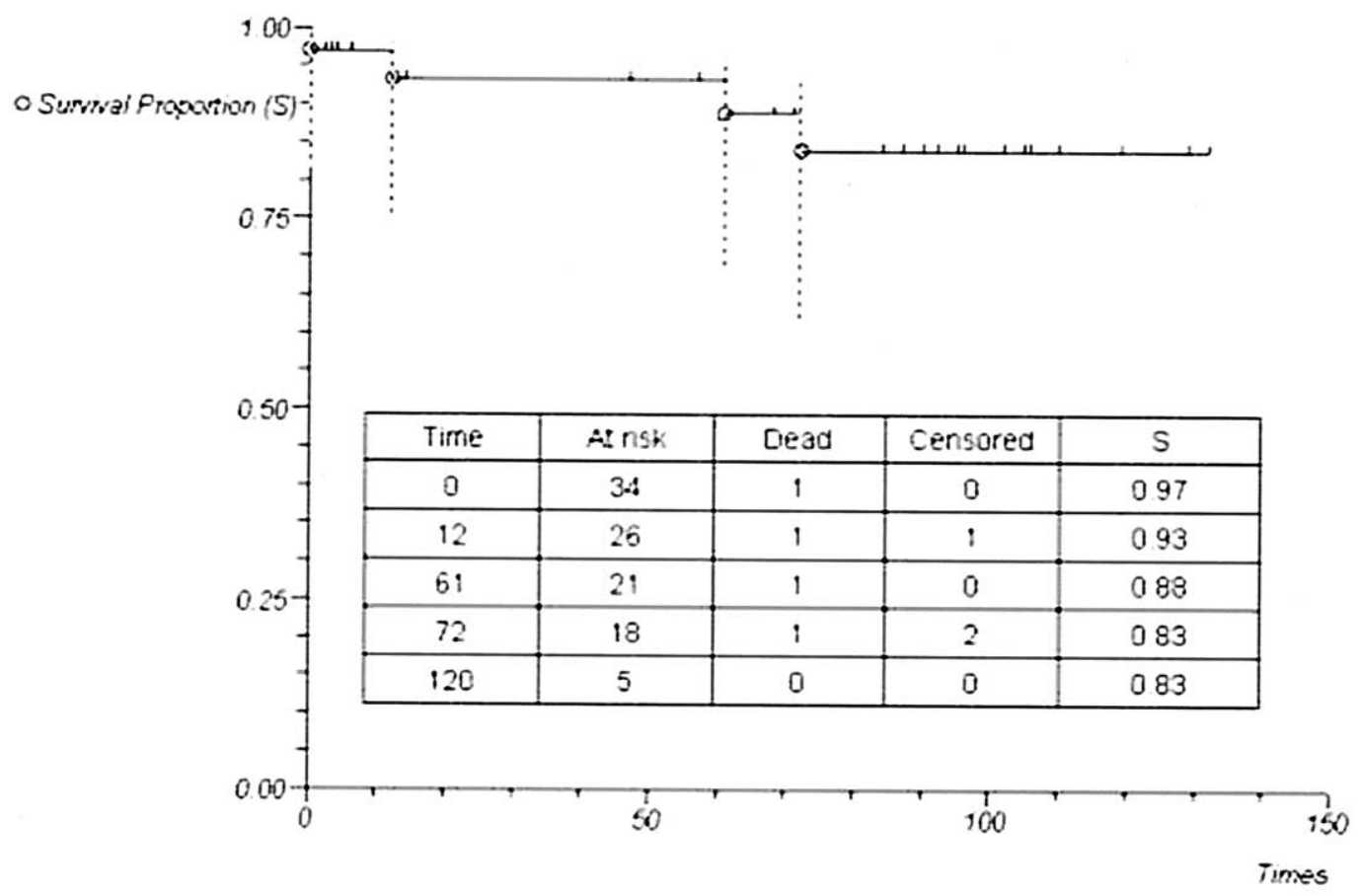

Figure 2. Cumulative survival (Kaplan-Meier method) for 34 patients in entire series who underwent aortic resection and end-to-end anastomosis. Major vertical lines indicate $95 \%$ confidence intervals of proportion.

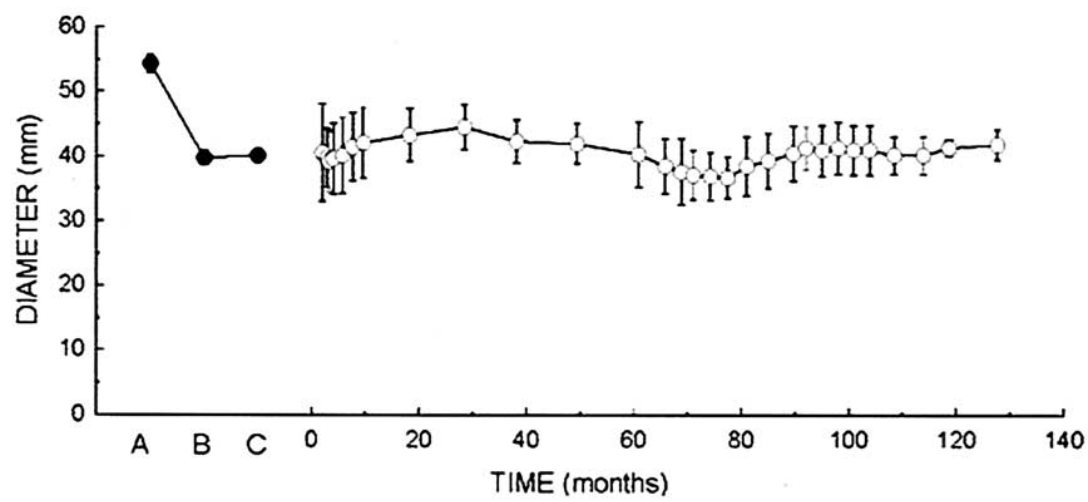

Figure 3. Mean aortic diameter, using all available measurements of 34 patients, before CPB (A), after CPB ( $B, 34$ patients), at discharge (C, 33 patients), and at follow-up (end of follow-up, 29 patients). For measurements at follow-up, moving band method was used. Mean aortic diameter is given with $70 \%$ confidence interval (error bars).

cedure depend on the cause of the disease, the size and the extension of the lesion, and the patient's profile and preferences. In presence of a dilated ascending aorta associated with a diseased aortic valve, the conventional techniques range from less radical procedures, such as supported and unsupported aortoplasty, ${ }^{13}$ to more aggressive interventions, including the separate replacement of the aortic valve and ascending aorta, valve-sparing root replacement, composite root replacement as described by Bentall and De
Bono, ${ }^{14}$ and aortic root replacement with autografts, homografts, or xenografts.

Aortic interposition grafting is virtually always required after resection of ascending and descending thoracic aortic aneurysms because of the necessity to reconstitute the aortic continuity without tension in patients with acquired or constitutional weak aortic wall. Actually, primary anastomosis for the repair of the aorta is considered the standard treatment for certain congenital anomalies, such as coarctation 


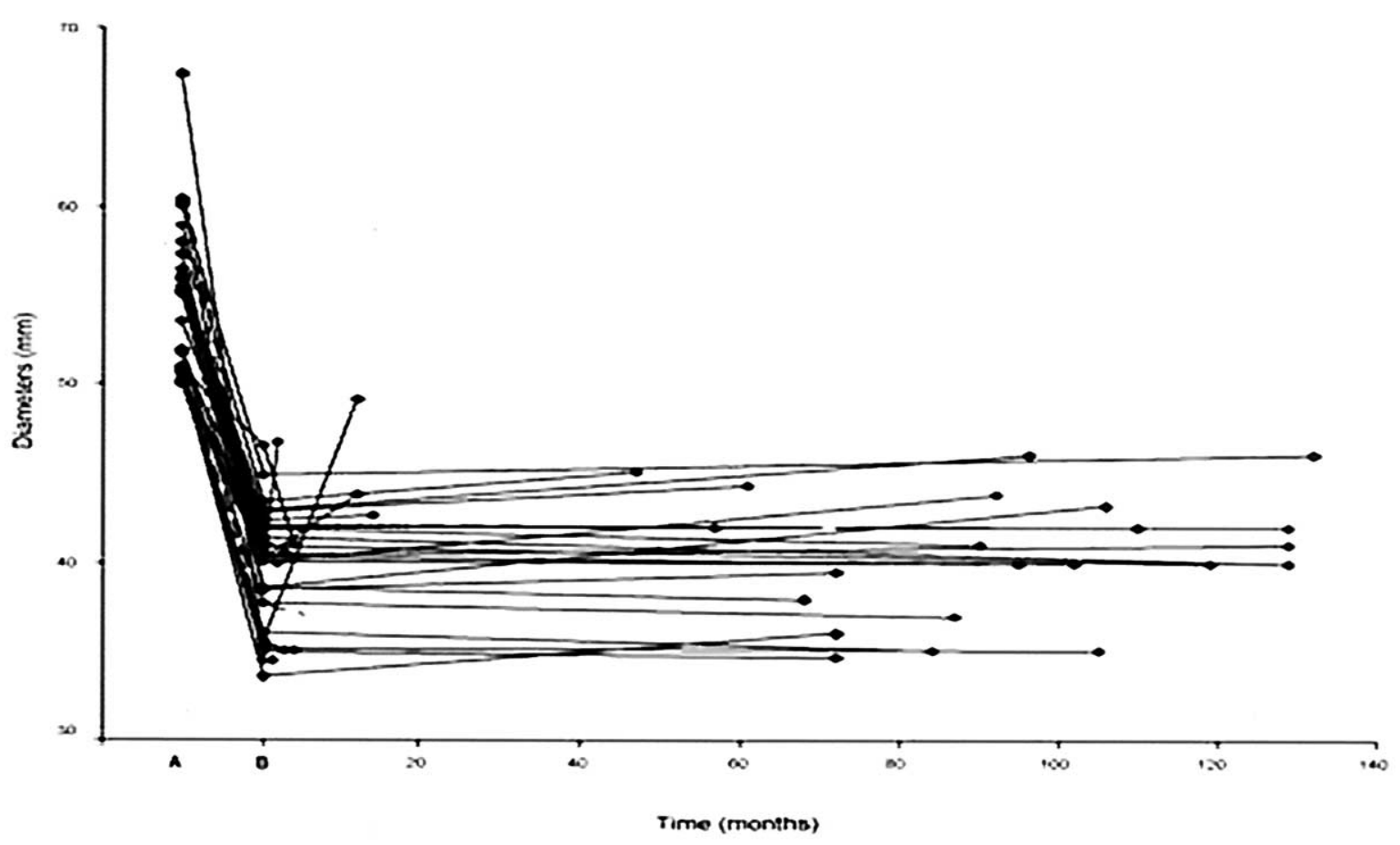

Figure 4. Individual increases in aortic diameter of patients with 2 or more measurements available before CPB $(A)$, after CPB $(B)$, and at follow-up.

and interrupted aortic arch, and it is occasionally used to repair traumatic aortic injuries. ${ }^{1}$ The aortic aneurysmal resection and end-to-end anastomosis technique has been used sporadically by other groups; however, there is a lack of information about its long-term durability. Recently, 10 patients who underwent primary end-to-end aortic anastomosis for ascending aneurysm were reported on in abstract form by Zher and associates ${ }^{15}$; these anastomoses were performed as a concomitant procedure in 8 cases, Within the average follow-up interval of 11 months (maximal follow-up 25 months), no treatment failures occurred. Vigano and coworkers ${ }^{3}$ reported their experience of this technique in 45 patients; during the follow-up period $(23.7 \pm 12.3$ months), 1 patient required reoperation and 2 patients died of unrelated causes. The surviving patients showed a low redilatation rate (1 patient, $2.3 \%$ ) and no incidence of pseudoaneurysm.

The presented series has the longest mean follow-up period of the literature and the long term satisfactory results confirm the effectiveness of the procedure and its durability. We underline, as a technical key point of this operation, the extensive dissection of pericardial reflections, freeing both the heart and the aortic arch. This enables relief of the tension on the anastomosis, thus making possible wide and radical resections.

This technique should be included among aortoplasty procedures, the most famous and commonly performed of which is that introduced early in the 1970s by Robicsek and colleagues. ${ }^{13}$ This technique, at long-term follow-up, has shown results comparable to or even better than those of radical aortic root replacement. ${ }^{15}$ However, a recent arti$\mathrm{cle}^{16}$ indicates better results of aortoplasty in those patients with aortic valve stenosis and poorer outcome in patients with dilatation of the ascending aorta associated with aortic valve regurgitation. The existence of defects in the aortic tissue structure in patients with aortic regurgitation has been suggested to explain these different behaviors. In our series we found, with regard to aortic score, no difference between patients with aortic regurgitation and those with stenosis (median difference 0,2 -sided $P=.57$ by Mann-Whitney $U$ test).

In contrast to this finding, none of our patients with aortic regurgitation exhibited recurrence of the ascending aorta aneurysm requiring reoperation. Actually, in our series the procedure was associated in most cases (26 patients) with a prosthetic aortic valve replacement for native aortic valve dysfunction. In our experience 2 patients had the stigmata of connective tissue disorders, although they did not fulfill the classic criteria of Marfan syndrome ${ }^{17}$; none of these subjects showed a redilatation of the aortic root during the follow-up. However, in light of the new insight about the management of patients with Marfan syndrome, which entails a more radical treatment extended to the aortic root, we no longer consider these patients with inherited systemic 
connective tissue diseases as potential candidates for this operation.

\section{Conclusions}

In light of the long-term results presented here, ascending aorta aneurysmal resection and primary end-to-end anastomosis represents an effective treatment in patients with ascending aortic aneurysms associated with valvular disease. Patients with Marfan syndrome or other inherited connective tissue disorders should be not considered for this treatment. The excellent results of the procedure suggest its use as an alternative to aortic interposition grafting in patients with aortic regurgitation, in whom a high recurrence rate of aneurysms with other techniques of aortoplasty is reported.

\section{References}

1. Stemmed CA, Wood MN, Razor AJ, Killeen JD, Gan KA. Primary repair of traumatic aortic rupture: a preferred approach. J Trauma. 1992;32:588-92

2. Ikonomidis JS, De Anda A, Miller DC. Resection of ascending aortic aneurysm without use of an interposition aortic graft. J Thorac Cardiovasc Surg. 2001;122:395-7.

3. Vigano M, Rinaldi M, D'Armini AM, Boffini M, Zattera GF, Alloni A, et al. Ascending aortic aneurysms treated by cuneiform resection and end-to-end anastomosis through a ministernotomy. Ann Thorac Surg. 2002;74:S1789-99.

4. Olinger GN, Schweiger JA, Galbraith TA. Primary repair of acute ascending aortic dissection. Ann Thorac Surg. 1987;44:389-93.

5. Pugilese P, Pessotto R, Santini F, Montalbano G, Luciani GB, Mazzucco A. Risk of late operations in patients with acute type A aortic dissection: impact of a more radical surgical approach. Eur J Cardiothoracic Surg. 1998;13:576-80.
6. Matthias Bechtel JF, Noack F, Sayk F, Erasmi AW, Bartels C, Sievers $\mathrm{HH}$. Histopathological grading of ascending aortic aneurysm: comparison of patients with bicuspid versus tricuspid aortic valve. $J$ Heart Valve Dis. 2003;12:54-9.

7. Kendall MG. Moving averages. In: Kendall MG, editor. Time series. London: Giffin; 1973. p. 47-54.

8. Pesarin F. Permutation testing for repeated measurements. In: Pesarin F, editor. Multivariate permutation tests with applications in biostatistics. 1st ed. New York: John Wiley; 2001.

9. de Sa M, Moshkovitz Y, Butany J, David TE. Histologic abnormalities of the ascending aorta and pulmonary trunk in patients with bicuspid aortic valve disease: clinical relevance to the Ross procedure. J Thorac Cardiovasc Surg. 1999;118:588-94.

10. Matthias Bechtel JF, Noack F, Sayk F, Erasmi AW, Bartels C, Sievers $\mathrm{HH}$. Histopathological grading of ascending aortic aneurysm: comparison of patients with bicuspid versus tricuspid aortic valve. J Heart Valve Dis. 2003;12:54-9.

11. Fedak PW, de Sa MP, Verma S, Nili N, Kazemian P, Butany J, et al. Vascular matrix remodeling in patients with bicuspid aortic valve malformations: implications for aortic dilatation. J Thorac Cardiovasc Surg. 2003;126:797-805.

12. Okamoto RJ, Xu H, Kouchoukos NT, Moon MR, Sundt TM. The influence of mechanical properties on wall stress and distensibility of the dilated ascending aorta. J Thorac Cardiovasc Surg. 2003;126:84250 .

13. Robicsek F, Thubrikar MJ. Conservative operation in the management of anular dilatation and ascending aortic aneurysm. Ann Thorac Surg. 1994;57:1672-4.

14. Bentall M, De Bono A. A technique for complete replacement of the ascending aorta. Thorax. 1968;23:338-9.

15. Zehr KJ, Sternik L, Schaff HV. Resection and primary end-to-end anastomosis of ascending aortic aneurysms. Circulation. 2000; 102(Suppl):II400-1.

16. Mueller XM, Tevaearai HT, Genton CY, Hurni M, Ruchat P, Fischer AP, et al. Drawback of aortoplasty for aneurysm of the ascending aorta associated with aortic valve disease. Ann Thorac Surg. 1997;63:762-7.

17. De Paepe A, Devereuz RB, Dietz HC, Hennekam RC, Pyeritz RE. Revised diagnostic criteria for the Marfan syndrome. Am J Med Genet. 1996;62:417-26. 